\title{
Crassocolides A-F, Cembranoids with a trans-Fused Lactone from the Soft Coral Sarcophyton crassocaule
}

Ho-Cheng Huang, ${ }^{\dagger, t}$ Atallah F. Ahmed, ${ }^{\dagger,}$ Jui-Hsin Su, ${ }^{\dagger}$ Chih-Hua Chao, ${ }^{\dagger}$ Yang-Chang Wu ${ }^{\S \S}$ Michael Y. Chiang, $\perp$ and Jyh-Horng Sheu ${ }^{*}+$

${ }^{\dagger}$ Department of Marine Biotechnology and Resources, National Sun Yat-sen University, Kaohsiung 804, Taiwan, ROC

\#Department of Chemical and Materials Engineering, Cheng Shiu University, Kaohsiung 833, Taiwan, ROC

${ }^{\S}$ Department of Pharmacognosy, Faculty of Pharmacy, Mansoura University, Mansoura 35516, Egypt

${ }^{\S \S}$ Graduate Institute of Natural Products, Kaohsiung Medical University, Kaohsiung 807, Taiwan, $R O C$

$\perp$ Department of Chemistry, National Sun Yat-sen University, Kaohsiung 804, Taiwan, ROC

*To whom correspondence should be addressed. Tel.: 886-7-5252000 ext. 5030, Fax: 886-7-5255020. E-mail: sheu@ mail.nsysu.edu.tw 
Figure 5. X-ray ORTEP diagram of crassocolide E (5)

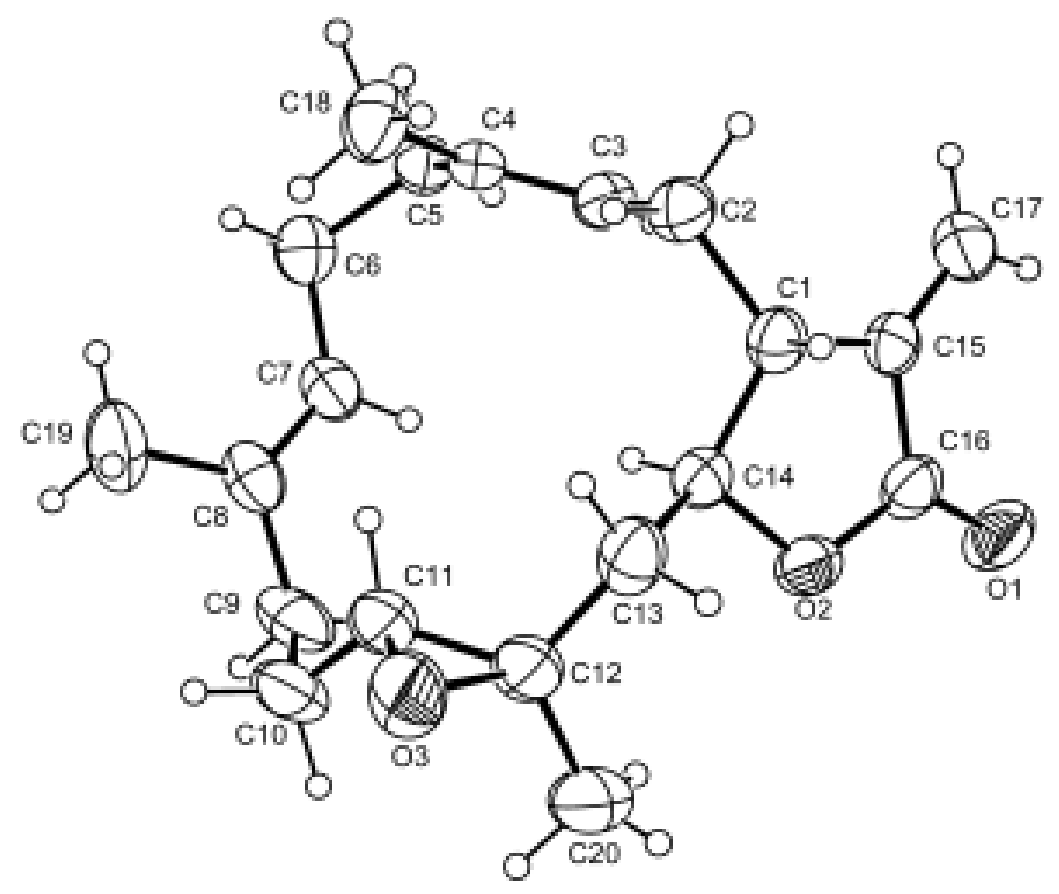


Table 1. Crystal data and structure refinement for crassocolide E (5).

Identification code

Empirical formula

Formula weight

Temperature

Wavelength

Crystal system, space group

Unit cell dimensions

Volume

Z, Calculated density

Absorption coefficient

$\mathrm{F}(000)$

Crystal size

Theta range for data collection

Limiting indices

Reflections collected / unique

Completeness to theta $=26.01$

Absorption correction

Max. and min. transmission

Refinement method

Data / restraints / parameters

Goodness-of-fit on $\mathrm{F}^{\wedge} 2$

Final $\mathrm{R}$ indices [I $>2 \operatorname{sigma}(\mathrm{I})]$

$\mathrm{R}$ indices (all data)

Absolute structure parameter

Largest diff. peak and hole crassocolide E (5)

$\mathrm{C}_{20} \mathrm{H}_{28} \mathrm{O}_{3}$

316.42

298(2) K

$0.71073 \mathrm{~A}$

Monoclinic, P 21

$\mathrm{a}=6.2085(12) \mathrm{A} \quad$ alpha $=90 \mathrm{deg}$.

$\mathrm{b}=9.7196(19) \mathrm{A} \quad$ beta $=95.40(3) \mathrm{deg}$.

$\mathrm{c}=15.606(3) \mathrm{A} \quad \mathrm{gamma}=90 \mathrm{deg}$.

937.5(3) $\mathrm{A}^{\wedge} 3$

2, $\quad 1.121 \mathrm{Mg} / \mathrm{m}^{\wedge} 3$

$0.074 \mathrm{~mm}^{\wedge}-1$

344

$0.65 \times 0.5 \times 0.4 \mathrm{~mm}$

2.47 to $26.01 \mathrm{deg}$.

$-7<=\mathrm{h}<=7,-11<=\mathrm{k}<=0,0<=\mathrm{l}<=19$

$2030 / 1960[\mathrm{R}(\mathrm{int})=0.0244]$

$99.9 \%$

Psi-scan

0.998 and 0.896

Full-matrix least-squares on $\mathrm{F}^{\wedge} 2$

$1960 / 1 / 211$

1.026

$\mathrm{R} 1=0.0412, \mathrm{wR} 2=0.1004$

$\mathrm{R} 1=0.0923, \mathrm{wR} 2=0.1199$

$-1(2)$

0.112 and -0.150 e. $\mathrm{A}^{\wedge}-3$ 
Table 2. Atomic coordinates ( $\mathrm{x} 10^{\wedge} 4$ ) and equivalent isotropic displacement arameters $\left(\mathrm{A}^{\wedge} 2 \times 10^{\wedge} 3\right)$ for crassocolide $\mathrm{E}(\mathbf{5})$. $\mathrm{U}(\mathrm{eq})$ is defined as one third of the trace of the orthogonalized Uij tensor.

\begin{tabular}{|c|c|c|c|c|}
\hline & $\mathrm{x}$ & $\mathrm{y}$ & $\mathrm{z}$ & $\mathrm{U}(\mathrm{eq})$ \\
\hline $\mathrm{O}(1)$ & $-3872(4)$ & $-3544(3)$ & $1183(2)$ & $101(1)$ \\
\hline $\mathrm{O}(2)$ & $-963(4)$ & $-2485(2)$ & $1819(2)$ & $75(1)$ \\
\hline $\mathrm{O}(3)$ & $5312(4)$ & $-896(3)$ & $3200(2)$ & $108(1)$ \\
\hline $\mathrm{C}(1)$ & $-528(5)$ & $-657(3)$ & $842(2)$ & $64(1)$ \\
\hline$C(2)$ & $-573(6)$ & 911(3) & $729(2)$ & $73(1)$ \\
\hline$C(3)$ & $-1935(5)$ & $1636(3)$ & $1322(2)$ & $64(1)$ \\
\hline $\mathrm{C}(4)$ & $-1508(5)$ & 2794(3) & $1754(2)$ & $58(1)$ \\
\hline$C(5)$ & $-3059(5)$ & $3381(4)$ & $2336(2)$ & $70(1)$ \\
\hline$C(6)$ & $-2080(5)$ & $3594(4)$ & $3262(2)$ & $76(1)$ \\
\hline$C(7)$ & $-1282(5)$ & 2292(4) & $3669(2)$ & $71(1)$ \\
\hline $\mathrm{C}(8)$ & $568(5)$ & 2053(4) & $4153(2)$ & $74(1)$ \\
\hline $\mathrm{C}(9)$ & $1036(7)$ & $663(5)$ & $4536(2)$ & $96(1)$ \\
\hline $\mathrm{C}(10)$ & $3239(7)$ & $36(6)$ & $4374(3)$ & $97(1)$ \\
\hline $\mathrm{C}(11)$ & $3471(6)$ & $-142(4)$ & $3439(3)$ & $79(1)$ \\
\hline$C(12)$ & $3151(6)$ & $-1420(4)$ & $2955(3)$ & $86(1)$ \\
\hline $\mathrm{C}(13)$ & $2496(5)$ & $-1309(4)$ & $2010(3)$ & $81(1)$ \\
\hline $\mathrm{C}(14)$ & $93(5)$ & $-1149(3)$ & 1761(2) & $63(1)$ \\
\hline$C(15)$ & $-2635(5)$ & $-1396(3)$ & $621(2)$ & $63(1)$ \\
\hline$C(16)$ & $-2630(6)$ & $-2585(4)$ & $1200(3)$ & $70(1)$ \\
\hline $\mathrm{C}(17)$ & $-4252(6)$ & $-1133(4)$ & $46(2)$ & $82(1)$ \\
\hline $\mathrm{C}(18)$ & $516(7)$ & $3607(5)$ & $1700(3)$ & $90(1)$ \\
\hline $\mathrm{C}(19)$ & $2247(6)$ & $3127(6)$ & $4378(3)$ & $104(2)$ \\
\hline$C(20)$ & $2660(11)$ & $-2765(5)$ & $3367(4)$ & $145(2)$ \\
\hline
\end{tabular}


Table 3. Bond lengths [A] and angles [deg] for crassocolide E (5).

\begin{tabular}{|c|c|}
\hline $\mathrm{O}(1)-\mathrm{C}(16)$ & $1.208(4)$ \\
\hline $\mathrm{O}(2)-\mathrm{C}(16)$ & $1.351(4)$ \\
\hline $\mathrm{O}(2)-\mathrm{C}(14)$ & $1.461(4)$ \\
\hline $\mathrm{O}(3)-\mathrm{C}(11)$ & $1.436(5)$ \\
\hline $\mathrm{O}(3)-\mathrm{C}(12)$ & $1.452(4)$ \\
\hline$C(1)-C(15)$ & $1.503(5)$ \\
\hline$C(1)-C(14)$ & $1.527(5)$ \\
\hline $\mathrm{C}(1)-\mathrm{C}(2)$ & $1.534(4)$ \\
\hline $\mathrm{C}(1)-\mathrm{H}(1)$ & 0.9800 \\
\hline$C(2)-C(3)$ & $1.488(5)$ \\
\hline $\mathrm{C}(2)-\mathrm{H}(2 \mathrm{~A})$ & 0.9700 \\
\hline $\mathrm{C}(2)-\mathrm{H}(2 \mathrm{~B})$ & 0.9700 \\
\hline$C(3)-C(4)$ & $1.327(5)$ \\
\hline $\mathrm{C}(3)-\mathrm{H}(3)$ & 0.9300 \\
\hline $\mathrm{C}(4)-\mathrm{C}(18)$ & $1.494(5)$ \\
\hline$C(4)-C(5)$ & $1.497(5)$ \\
\hline$C(5)-C(6)$ & $1.528(4)$ \\
\hline $\mathrm{C}(5)-\mathrm{H}(5 \mathrm{~A})$ & 0.9700 \\
\hline $\mathrm{C}(5)-\mathrm{H}(5 \mathrm{~B})$ & 0.9700 \\
\hline$C(6)-C(7)$ & $1.480(5)$ \\
\hline $\mathrm{C}(6)-\mathrm{H}(6 \mathrm{~A})$ & 0.9700 \\
\hline $\mathrm{C}(6)-\mathrm{H}(6 \mathrm{~B})$ & 0.9700 \\
\hline$C(7)-C(8)$ & $1.334(5)$ \\
\hline $\mathrm{C}(7)-\mathrm{H}(7)$ & 0.9300 \\
\hline $\mathrm{C}(8)-\mathrm{C}(19)$ & $1.493(6)$ \\
\hline C(8)-C(9) & $1.495(6)$ \\
\hline $\mathrm{C}(9)-\mathrm{C}(10)$ & $1.540(6)$ \\
\hline $\mathrm{C}(9)-\mathrm{H}(9 \mathrm{~A})$ & 0.9700 \\
\hline $\mathrm{C}(9)-\mathrm{H}(9 \mathrm{~B})$ & 0.9700 \\
\hline $\mathrm{C}(10)-\mathrm{C}(11)$ & $1.490(6)$ \\
\hline $\mathrm{C}(10)-\mathrm{H}(10 \mathrm{~A})$ & 0.9700 \\
\hline $\mathrm{C}(10)-\mathrm{H}(10 \mathrm{~B})$ & 0.9700 \\
\hline$C(11)-C(12)$ & $1.458(6)$ \\
\hline $\mathrm{C}(11)-\mathrm{H}(11)$ & 0.9800 \\
\hline $\mathrm{C}(12)-\mathrm{C}(13)$ & $1.497(5)$ \\
\hline$C(12)-C(20)$ & $1.500(6)$ \\
\hline$C(13)-C(14)$ & $1.513(4)$ \\
\hline $\mathrm{C}(13)-\mathrm{H}(13 \mathrm{~A})$ & 0.9700 \\
\hline $\mathrm{C}(13)-\mathrm{H}(13 \mathrm{~B})$ & 0.9700 \\
\hline
\end{tabular}




\begin{tabular}{|c|c|}
\hline $\mathrm{C}(14)-\mathrm{H}(14)$ & 0.9800 \\
\hline $\mathrm{C}(15)-\mathrm{C}(17)$ & $1.308(4)$ \\
\hline$C(15)-C(16)$ & $1.466(5)$ \\
\hline $\mathrm{C}(17)-\mathrm{H}(17 \mathrm{~A})$ & 0.9300 \\
\hline $\mathrm{C}(17)-\mathrm{H}(17 \mathrm{~B})$ & 0.9300 \\
\hline $\mathrm{C}(18)-\mathrm{H}(18 \mathrm{~A})$ & 0.9600 \\
\hline $\mathrm{C}(18)-\mathrm{H}(18 \mathrm{~B})$ & 0.9600 \\
\hline $\mathrm{C}(18)-\mathrm{H}(18 \mathrm{C})$ & 0.9600 \\
\hline $\mathrm{C}(19)-\mathrm{H}(19 \mathrm{~A})$ & 0.9600 \\
\hline C(19)-H(19B) & 0.9600 \\
\hline C(19)-H(19C) & 0.9600 \\
\hline $\mathrm{C}(20)-\mathrm{H}(20 \mathrm{~A})$ & 0.9600 \\
\hline $\mathrm{C}(20)-\mathrm{H}(20 \mathrm{~B})$ & 0.9600 \\
\hline $\mathrm{C}(20)-\mathrm{H}(20 \mathrm{C})$ & 0.9600 \\
\hline $\mathrm{C}(16)-\mathrm{O}(2)-\mathrm{C}(14)$ & $109.8(3)$ \\
\hline $\mathrm{C}(11)-\mathrm{O}(3)-\mathrm{C}(12)$ & $60.6(2)$ \\
\hline$C(15)-C(1)-C(14)$ & $101.7(3)$ \\
\hline $\mathrm{C}(15)-\mathrm{C}(1)-\mathrm{C}(2)$ & $116.3(3)$ \\
\hline $\mathrm{C}(14)-\mathrm{C}(1)-\mathrm{C}(2)$ & $114.8(3)$ \\
\hline $\mathrm{C}(15)-\mathrm{C}(1)-\mathrm{H}(1)$ & 107.9 \\
\hline $\mathrm{C}(14)-\mathrm{C}(1)-\mathrm{H}(1)$ & 107.9 \\
\hline $\mathrm{C}(2)-\mathrm{C}(1)-\mathrm{H}(1)$ & 107.9 \\
\hline $\mathrm{C}(3)-\mathrm{C}(2)-\mathrm{C}(1)$ & $113.9(3)$ \\
\hline $\mathrm{C}(3)-\mathrm{C}(2)-\mathrm{H}(2 \mathrm{~A})$ & 108.8 \\
\hline $\mathrm{C}(1)-\mathrm{C}(2)-\mathrm{H}(2 \mathrm{~A})$ & 108.8 \\
\hline $\mathrm{C}(3)-\mathrm{C}(2)-\mathrm{H}(2 \mathrm{~B})$ & 108.8 \\
\hline $\mathrm{C}(1)-\mathrm{C}(2)-\mathrm{H}(2 \mathrm{~B})$ & 108.8 \\
\hline $\mathrm{H}(2 \mathrm{~A})-\mathrm{C}(2)-\mathrm{H}(2 \mathrm{~B})$ & 107.7 \\
\hline $\mathrm{C}(4)-\mathrm{C}(3)-\mathrm{C}(2)$ & $128.3(3)$ \\
\hline $\mathrm{C}(4)-\mathrm{C}(3)-\mathrm{H}(3)$ & 115.8 \\
\hline $\mathrm{C}(2)-\mathrm{C}(3)-\mathrm{H}(3)$ & 115.8 \\
\hline $\mathrm{C}(3)-\mathrm{C}(4)-\mathrm{C}(18)$ & $123.3(3)$ \\
\hline$C(3)-C(4)-C(5)$ & $121.6(3)$ \\
\hline $\mathrm{C}(18)-\mathrm{C}(4)-\mathrm{C}(5)$ & $115.1(3)$ \\
\hline$C(4)-C(5)-C(6)$ & $113.8(3)$ \\
\hline $\mathrm{C}(4)-\mathrm{C}(5)-\mathrm{H}(5 \mathrm{~A})$ & 108.8 \\
\hline $\mathrm{C}(6)-\mathrm{C}(5)-\mathrm{H}(5 \mathrm{~A})$ & 108.8 \\
\hline $\mathrm{C}(4)-\mathrm{C}(5)-\mathrm{H}(5 \mathrm{~B})$ & 108.8 \\
\hline $\mathrm{C}(6)-\mathrm{C}(5)-\mathrm{H}(5 \mathrm{~B})$ & 108.8 \\
\hline $\mathrm{H}(5 \mathrm{~A})-\mathrm{C}(5)-\mathrm{H}(5 \mathrm{~B})$ & 107.7 \\
\hline
\end{tabular}




\begin{tabular}{|c|c|}
\hline$C(7)-C(6)-C(5)$ & $112.2(3)$ \\
\hline $\mathrm{C}(7)-\mathrm{C}(6)-\mathrm{H}(6 \mathrm{~A})$ & 109.2 \\
\hline $\mathrm{C}(5)-\mathrm{C}(6)-\mathrm{H}(6 \mathrm{~A})$ & 109.2 \\
\hline $\mathrm{C}(7)-\mathrm{C}(6)-\mathrm{H}(6 \mathrm{~B})$ & 109.2 \\
\hline $\mathrm{C}(5)-\mathrm{C}(6)-\mathrm{H}(6 \mathrm{~B})$ & 109.2 \\
\hline$H(6 A)-C(6)-H(6 B)$ & 107.9 \\
\hline$C(8)-C(7)-C(6)$ & $128.8(3)$ \\
\hline $\mathrm{C}(8)-\mathrm{C}(7)-\mathrm{H}(7)$ & 115.6 \\
\hline $\mathrm{C}(6)-\mathrm{C}(7)-\mathrm{H}(7)$ & 115.6 \\
\hline $\mathrm{C}(7)-\mathrm{C}(8)-\mathrm{C}(19)$ & $123.7(4)$ \\
\hline $\mathrm{C}(7)-\mathrm{C}(8)-\mathrm{C}(9)$ & $120.6(3)$ \\
\hline $\mathrm{C}(19)-\mathrm{C}(8)-\mathrm{C}(9)$ & $115.7(3)$ \\
\hline $\mathrm{C}(8)-\mathrm{C}(9)-\mathrm{C}(10)$ & $115.7(4)$ \\
\hline $\mathrm{C}(8)-\mathrm{C}(9)-\mathrm{H}(9 \mathrm{~A})$ & 108.3 \\
\hline $\mathrm{C}(10)-\mathrm{C}(9)-\mathrm{H}(9 \mathrm{~A})$ & 108.3 \\
\hline $\mathrm{C}(8)-\mathrm{C}(9)-\mathrm{H}(9 \mathrm{~B})$ & 108.3 \\
\hline $\mathrm{C}(10)-\mathrm{C}(9)-\mathrm{H}(9 \mathrm{~B})$ & 108.3 \\
\hline $\mathrm{H}(9 \mathrm{~A})-\mathrm{C}(9)-\mathrm{H}(9 \mathrm{~B})$ & 107.4 \\
\hline $\mathrm{C}(11)-\mathrm{C}(10)-\mathrm{C}(9)$ & $112.1(3)$ \\
\hline $\mathrm{C}(11)-\mathrm{C}(10)-\mathrm{H}(10 \mathrm{~A})$ & 109.2 \\
\hline $\mathrm{C}(9)-\mathrm{C}(10)-\mathrm{H}(10 \mathrm{~A})$ & 109.2 \\
\hline $\mathrm{C}(11)-\mathrm{C}(10)-\mathrm{H}(10 \mathrm{~B})$ & 109.2 \\
\hline $\mathrm{C}(9)-\mathrm{C}(10)-\mathrm{H}(10 \mathrm{~B})$ & 109.2 \\
\hline $\mathrm{H}(10 \mathrm{~A})-\mathrm{C}(10)-\mathrm{H}(10 \mathrm{~B})$ & 107.9 \\
\hline $\mathrm{O}(3)-\mathrm{C}(11)-\mathrm{C}(12)$ & $60.2(3)$ \\
\hline $\mathrm{O}(3)-\mathrm{C}(11)-\mathrm{C}(10)$ & $117.8(3)$ \\
\hline$C(12)-C(11)-C(10)$ & $125.8(4)$ \\
\hline $\mathrm{O}(3)-\mathrm{C}(11)-\mathrm{H}(11)$ & 114.0 \\
\hline $\mathrm{C}(12)-\mathrm{C}(11)-\mathrm{H}(11)$ & 114.0 \\
\hline $\mathrm{C}(10)-\mathrm{C}(11)-\mathrm{H}(11)$ & 114.0 \\
\hline $\mathrm{O}(3)-\mathrm{C}(12)-\mathrm{C}(11)$ & $59.2(2)$ \\
\hline $\mathrm{O}(3)-\mathrm{C}(12)-\mathrm{C}(13)$ & $113.1(3)$ \\
\hline $\mathrm{C}(11)-\mathrm{C}(12)-\mathrm{C}(13)$ & $117.4(4)$ \\
\hline $\mathrm{O}(3)-\mathrm{C}(12)-\mathrm{C}(20)$ & $114.4(4)$ \\
\hline $\mathrm{C}(11)-\mathrm{C}(12)-\mathrm{C}(20)$ & $123.0(4)$ \\
\hline$C(13)-C(12)-C(20)$ & $115.9(4)$ \\
\hline$C(12)-C(13)-C(14)$ & $115.5(3)$ \\
\hline $\mathrm{C}(12)-\mathrm{C}(13)-\mathrm{H}(13 \mathrm{~A})$ & 108.4 \\
\hline $\mathrm{C}(14)-\mathrm{C}(13)-\mathrm{H}(13 \mathrm{~A})$ & 108.4 \\
\hline $\mathrm{C}(12)-\mathrm{C}(13)-\mathrm{H}(13 \mathrm{~B})$ & 108.4 \\
\hline $\mathrm{C}(14)-\mathrm{C}(13)-\mathrm{H}(13 \mathrm{~B})$ & 108.4 \\
\hline
\end{tabular}




\begin{tabular}{|c|c|}
\hline $\mathrm{H}(13 \mathrm{~A})-\mathrm{C}(13)-\mathrm{H}(13 \mathrm{~B})$ & 107.5 \\
\hline $\mathrm{O}(2)-\mathrm{C}(14)-\mathrm{C}(13)$ & $109.3(3)$ \\
\hline $\mathrm{O}(2)-\mathrm{C}(14)-\mathrm{C}(1)$ & $105.1(2)$ \\
\hline$C(13)-C(14)-C(1)$ & $115.4(3)$ \\
\hline $\mathrm{O}(2)-\mathrm{C}(14)-\mathrm{H}(14)$ & 108.9 \\
\hline $\mathrm{C}(13)-\mathrm{C}(14)-\mathrm{H}(14)$ & 108.9 \\
\hline $\mathrm{C}(1)-\mathrm{C}(14)-\mathrm{H}(14)$ & 108.9 \\
\hline$C(17)-C(15)-C(16)$ & $122.3(3)$ \\
\hline $\mathrm{C}(17)-\mathrm{C}(15)-\mathrm{C}(1)$ & $131.2(3)$ \\
\hline$C(16)-C(15)-C(1)$ & $106.5(3)$ \\
\hline $\mathrm{O}(1)-\mathrm{C}(16)-\mathrm{O}(2)$ & $121.0(3)$ \\
\hline $\mathrm{O}(1)-\mathrm{C}(16)-\mathrm{C}(15)$ & $129.1(3)$ \\
\hline $\mathrm{O}(2)-\mathrm{C}(16)-\mathrm{C}(15)$ & 109.9(3) \\
\hline $\mathrm{C}(15)-\mathrm{C}(17)-\mathrm{H}(17 \mathrm{~A})$ & 120.0 \\
\hline $\mathrm{C}(15)-\mathrm{C}(17)-\mathrm{H}(17 \mathrm{~B})$ & 120.0 \\
\hline $\mathrm{H}(17 \mathrm{~A})-\mathrm{C}(17)-\mathrm{H}(17 \mathrm{~B})$ & 120.0 \\
\hline $\mathrm{C}(4)-\mathrm{C}(18)-\mathrm{H}(18 \mathrm{~A})$ & 109.5 \\
\hline $\mathrm{C}(4)-\mathrm{C}(18)-\mathrm{H}(18 \mathrm{~B})$ & 109.5 \\
\hline $\mathrm{H}(18 \mathrm{~A})-\mathrm{C}(18)-\mathrm{H}(18 \mathrm{~B})$ & 109.5 \\
\hline $\mathrm{C}(4)-\mathrm{C}(18)-\mathrm{H}(18 \mathrm{C})$ & 109.5 \\
\hline $\mathrm{H}(18 \mathrm{~A})-\mathrm{C}(18)-\mathrm{H}(18 \mathrm{C})$ & 109.5 \\
\hline $\mathrm{H}(18 \mathrm{~B})-\mathrm{C}(18)-\mathrm{H}(18 \mathrm{C})$ & 109.5 \\
\hline $\mathrm{C}(8)-\mathrm{C}(19)-\mathrm{H}(19 \mathrm{~A})$ & 109.5 \\
\hline $\mathrm{C}(8)-\mathrm{C}(19)-\mathrm{H}(19 \mathrm{~B})$ & 109.5 \\
\hline $\mathrm{H}(19 \mathrm{~A})-\mathrm{C}(19)-\mathrm{H}(19 \mathrm{~B})$ & 109.5 \\
\hline $\mathrm{C}(8)-\mathrm{C}(19)-\mathrm{H}(19 \mathrm{C})$ & 109.5 \\
\hline $\mathrm{H}(19 \mathrm{~A})-\mathrm{C}(19)-\mathrm{H}(19 \mathrm{C})$ & 109.5 \\
\hline H(19B)-C(19)-H(19C) & 109.5 \\
\hline $\mathrm{C}(12)-\mathrm{C}(20)-\mathrm{H}(20 \mathrm{~A})$ & 109.5 \\
\hline $\mathrm{C}(12)-\mathrm{C}(20)-\mathrm{H}(20 \mathrm{~B})$ & 109.5 \\
\hline $\mathrm{H}(20 \mathrm{~A})-\mathrm{C}(20)-\mathrm{H}(20 \mathrm{~B})$ & 109.5 \\
\hline $\mathrm{C}(12)-\mathrm{C}(20)-\mathrm{H}(20 \mathrm{C})$ & 109.5 \\
\hline $\mathrm{H}(20 \mathrm{~A})-\mathrm{C}(20)-\mathrm{H}(20 \mathrm{C})$ & 109.5 \\
\hline $\mathrm{H}(20 \mathrm{~B})-\mathrm{C}(20)-\mathrm{H}(20 \mathrm{C})$ & 109.5 \\
\hline
\end{tabular}

Symmetry transformations used to generate equivalent atoms: 
Table 4. Anisotropic displacement parameters $\left(\mathrm{A}^{\wedge} 2 \times 10^{\wedge} 3\right)$ for crassocolide $\mathrm{E}(\mathbf{5})$.

The anisotropic displacement factor exponent takes the form:

$-2 \mathrm{pi}^{\wedge} 2\left[\mathrm{~h}^{\wedge} 2 \mathrm{a}^{* \wedge} 2 \mathrm{U} 11+\ldots+2 \mathrm{~h} \mathrm{k} \mathrm{a}^{*} \mathrm{~b}^{*} \mathrm{U} 12\right]$

\begin{tabular}{|c|c|c|c|c|c|c|}
\hline & U11 & $\mathrm{U} 22$ & U33 & $\mathrm{U} 23$ & U13 & U12 \\
\hline $\mathrm{O}(1)$ & $95(2)$ & $59(2)$ & $142(3)$ & $1(2)$ & $-19(2)$ & $-12(2)$ \\
\hline $\mathrm{O}(2)$ & $72(1)$ & $57(1)$ & $93(2)$ & $9(1)$ & $-9(1)$ & $-1(1)$ \\
\hline $\mathrm{O}(3)$ & $61(1)$ & 101(2) & $155(3)$ & $-16(2)$ & $-27(2)$ & $7(2)$ \\
\hline $\mathrm{C}(1)$ & $71(2)$ & $57(2)$ & $64(2)$ & $-9(2)$ & $9(2)$ & $3(2)$ \\
\hline $\mathrm{C}(2)$ & $87(2)$ & $64(2)$ & $67(2)$ & $3(2)$ & $11(2)$ & $-6(2)$ \\
\hline $\mathrm{C}(3)$ & $67(2)$ & $53(2)$ & $70(2)$ & $5(2)$ & $-1(2)$ & $1(2)$ \\
\hline $\mathrm{C}(4)$ & $69(2)$ & $49(2)$ & $52(2)$ & $8(1)$ & $-6(1)$ & $2(2)$ \\
\hline $\mathrm{C}(5)$ & $66(2)$ & $60(2)$ & $80(2)$ & $-1(2)$ & $-8(2)$ & $7(2)$ \\
\hline$C(6)$ & $71(2)$ & $82(3)$ & $74(2)$ & $-10(2)$ & $12(2)$ & $-3(2)$ \\
\hline$C(7)$ & $64(2)$ & $91(3)$ & $57(2)$ & $8(2)$ & $3(2)$ & $-20(2)$ \\
\hline$C(8)$ & $64(2)$ & 102(3) & $57(2)$ & $-6(2)$ & $6(2)$ & $-9(2)$ \\
\hline $\mathrm{C}(9)$ & $95(3)$ & 131(4) & $60(2)$ & $29(2)$ & $-6(2)$ & $-8(3)$ \\
\hline$C(10)$ & $91(3)$ & 107(3) & $86(3)$ & $26(3)$ & $-29(2)$ & $3(3)$ \\
\hline $\mathrm{C}(11)$ & $66(2)$ & $74(3)$ & $93(3)$ & $7(2)$ & $-17(2)$ & $1(2)$ \\
\hline$C(12)$ & $65(2)$ & $74(2)$ & 111(3) & $8(2)$ & $-26(2)$ & $3(2)$ \\
\hline$C(13)$ & $64(2)$ & $73(2)$ & 106(3) & $-14(2)$ & $-1(2)$ & $1(2)$ \\
\hline$C(14)$ & $66(2)$ & $54(2)$ & $68(2)$ & $-3(2)$ & $1(2)$ & $2(2)$ \\
\hline $\mathrm{C}(15)$ & $74(2)$ & $57(2)$ & $58(2)$ & $-13(2)$ & $3(2)$ & $8(2)$ \\
\hline$C(16)$ & $67(2)$ & $51(2)$ & $91(3)$ & $-8(2)$ & $-1(2)$ & $6(2)$ \\
\hline$C(17)$ & $93(3)$ & $81(2)$ & $69(2)$ & $-9(2)$ & $-5(2)$ & $6(2)$ \\
\hline $\mathrm{C}(18)$ & 104(3) & $85(3)$ & $84(3)$ & $-12(2)$ & $23(2)$ & $-28(3)$ \\
\hline$C(19)$ & $73(2)$ & $116(4)$ & 118(3) & $-39(3)$ & $-12(2)$ & $-4(2)$ \\
\hline$C(20)$ & $173(5)$ & $86(3)$ & $161(5)$ & $42(3)$ & $-70(4)$ & $-23(3)$ \\
\hline
\end{tabular}


Table 5. Hydrogen coordinates ( x 10^4) and isotropic displacement parameters $\left(A^{\wedge} 2 \times 10^{\wedge} 3\right)$ for crassocolide $E(5)$.

\begin{tabular}{|c|c|c|c|c|}
\hline & $\mathrm{x}$ & y & $\mathrm{z}$ & $\mathrm{U}(\mathrm{eq})$ \\
\hline $\mathrm{H}(1)$ & 531 & -1030 & 476 & 77 \\
\hline $\mathrm{H}(2 \mathrm{~A})$ & 895 & 1258 & 823 & 87 \\
\hline $\mathrm{H}(2 \mathrm{~B})$ & -1110 & 1125 & 141 & 87 \\
\hline $\mathrm{H}(3)$ & -3255 & 1224 & 1398 & 77 \\
\hline $\mathrm{H}(5 \mathrm{~A})$ & -4292 & 2769 & 2339 & 83 \\
\hline $\mathrm{H}(5 \mathrm{~B})$ & -3583 & 4258 & 2105 & 83 \\
\hline $\mathrm{H}(6 \mathrm{~A})$ & -890 & 4242 & 3266 & 91 \\
\hline $\mathrm{H}(6 \mathrm{~B})$ & -3165 & 3989 & 3597 & 91 \\
\hline $\mathrm{H}(7)$ & -2195 & 1538 & 3575 & 85 \\
\hline $\mathrm{H}(9 \mathrm{~A})$ & -94 & 37 & 4310 & 116 \\
\hline $\mathrm{H}(9 \mathrm{~B})$ & 960 & 725 & 5153 & 116 \\
\hline $\mathrm{H}(10 \mathrm{~A})$ & 4387 & 629 & 4625 & 117 \\
\hline $\mathrm{H}(10 \mathrm{~B})$ & 3394 & -852 & 4658 & 117 \\
\hline $\mathrm{H}(11)$ & 3144 & 689 & 3095 & 95 \\
\hline $\mathrm{H}(13 \mathrm{~A})$ & 2993 & -2125 & 1730 & 98 \\
\hline $\mathrm{H}(13 \mathrm{~B})$ & 3235 & -525 & 1788 & 98 \\
\hline $\mathrm{H}(14)$ & -505 & -510 & 2162 & 75 \\
\hline $\mathrm{H}(17 \mathrm{~A})$ & -5453 & -1709 & -7 & 98 \\
\hline $\mathrm{H}(17 \mathrm{~B})$ & -4201 & -369 & -311 & 98 \\
\hline $\mathrm{H}(18 \mathrm{~A})$ & 1347 & 3206 & 1276 & 135 \\
\hline $\mathrm{H}(18 \mathrm{~B})$ & 1352 & 3601 & 2250 & 135 \\
\hline $\mathrm{H}(18 \mathrm{C})$ & 149 & 4538 & 1540 & 135 \\
\hline $\mathrm{H}(19 \mathrm{~A})$ & 1770 & 3989 & 4127 & 155 \\
\hline $\mathrm{H}(19 \mathrm{~B})$ & 3579 & 2860 & 4159 & 155 \\
\hline $\mathrm{H}(19 \mathrm{C})$ & 2469 & 3222 & 4992 & 155 \\
\hline$H(20 A)$ & 1121 & -2882 & 3349 & 218 \\
\hline $\mathrm{H}(20 \mathrm{~B})$ & 3277 & -2769 & 3954 & 218 \\
\hline $\mathrm{H}(20 \mathrm{C})$ & 3267 & -3504 & 3060 & 218 \\
\hline
\end{tabular}


Table 6. Torsion angles [deg] for crassocolide E (5).

\begin{tabular}{|c|c|}
\hline$C(15)-C(1)-C(2)-C(3)$ & $65.2(4)$ \\
\hline $\mathrm{C}(14)-\mathrm{C}(1)-\mathrm{C}(2)-\mathrm{C}(3)$ & $-53.3(5)$ \\
\hline $\mathrm{C}(1)-\mathrm{C}(2)-\mathrm{C}(3)-\mathrm{C}(4)$ & $138.0(4)$ \\
\hline $\mathrm{C}(2)-\mathrm{C}(3)-\mathrm{C}(4)-\mathrm{C}(18)$ & $0.9(5)$ \\
\hline $\mathrm{C}(2)-\mathrm{C}(3)-\mathrm{C}(4)-\mathrm{C}(5)$ & $-179.3(3)$ \\
\hline$C(3)-C(4)-C(5)-C(6)$ & $123.3(4)$ \\
\hline$C(18)-C(4)-C(5)-C(6)$ & $-56.9(4)$ \\
\hline $\mathrm{C}(4)-\mathrm{C}(5)-\mathrm{C}(6)-\mathrm{C}(7)$ & $-59.8(4)$ \\
\hline$C(5)-C(6)-C(7)-C(8)$ & $136.1(4)$ \\
\hline $\mathrm{C}(6)-\mathrm{C}(7)-\mathrm{C}(8)-\mathrm{C}(19)$ & $-0.1(6)$ \\
\hline $\mathrm{C}(6)-\mathrm{C}(7)-\mathrm{C}(8)-\mathrm{C}(9)$ & $177.6(3)$ \\
\hline $\mathrm{C}(7)-\mathrm{C}(8)-\mathrm{C}(9)-\mathrm{C}(10)$ & $128.8(4)$ \\
\hline $\mathrm{C}(19)-\mathrm{C}(8)-\mathrm{C}(9)-\mathrm{C}(10)$ & $-53.4(5)$ \\
\hline $\mathrm{C}(8)-\mathrm{C}(9)-\mathrm{C}(10)-\mathrm{C}(11)$ & $-60.0(5)$ \\
\hline $\mathrm{C}(12)-\mathrm{O}(3)-\mathrm{C}(11)-\mathrm{C}(10)$ & $117.4(4)$ \\
\hline $\mathrm{C}(9)-\mathrm{C}(10)-\mathrm{C}(11)-\mathrm{O}(3)$ & $-171.2(4)$ \\
\hline $\mathrm{C}(9)-\mathrm{C}(10)-\mathrm{C}(11)-\mathrm{C}(12)$ & $-99.4(5)$ \\
\hline $\mathrm{C}(11)-\mathrm{O}(3)-\mathrm{C}(12)-\mathrm{C}(13)$ & $109.1(4)$ \\
\hline $\mathrm{C}(11)-\mathrm{O}(3)-\mathrm{C}(12)-\mathrm{C}(20)$ & $-115.2(5)$ \\
\hline $\mathrm{C}(10)-\mathrm{C}(11)-\mathrm{C}(12)-\mathrm{O}(3)$ & $-104.5(4)$ \\
\hline $\mathrm{O}(3)-\mathrm{C}(11)-\mathrm{C}(12)-\mathrm{C}(13)$ & $-101.8(4)$ \\
\hline $\mathrm{C}(10)-\mathrm{C}(11)-\mathrm{C}(12)-\mathrm{C}(13)$ & $153.7(3)$ \\
\hline $\mathrm{O}(3)-\mathrm{C}(11)-\mathrm{C}(12)-\mathrm{C}(20)$ & $100.8(5)$ \\
\hline $\mathrm{C}(10)-\mathrm{C}(11)-\mathrm{C}(12)-\mathrm{C}(20)$ & $-3.8(6)$ \\
\hline $\mathrm{O}(3)-\mathrm{C}(12)-\mathrm{C}(13)-\mathrm{C}(14)$ & $-151.0(3)$ \\
\hline $\mathrm{C}(11)-\mathrm{C}(12)-\mathrm{C}(13)-\mathrm{C}(14)$ & $-85.1(5)$ \\
\hline $\mathrm{C}(20)-\mathrm{C}(12)-\mathrm{C}(13)-\mathrm{C}(14)$ & $74.0(6)$ \\
\hline $\mathrm{C}(16)-\mathrm{O}(2)-\mathrm{C}(14)-\mathrm{C}(13)$ & $-145.8(3)$ \\
\hline $\mathrm{C}(16)-\mathrm{O}(2)-\mathrm{C}(14)-\mathrm{C}(1)$ & $-21.3(3)$ \\
\hline $\mathrm{C}(12)-\mathrm{C}(13)-\mathrm{C}(14)-\mathrm{O}(2)$ & $-77.4(4)$ \\
\hline $\mathrm{C}(12)-\mathrm{C}(13)-\mathrm{C}(14)-\mathrm{C}(1)$ & $164.3(3)$ \\
\hline $\mathrm{C}(15)-\mathrm{C}(1)-\mathrm{C}(14)-\mathrm{O}(2)$ & $26.0(3)$ \\
\hline
\end{tabular}




$\begin{array}{lr}\mathrm{C}(2)-\mathrm{C}(1)-\mathrm{C}(14)-\mathrm{O}(2) & 152.4(3) \\ \mathrm{C}(15)-\mathrm{C}(1)-\mathrm{C}(14)-\mathrm{C}(13) & 146.6(3) \\ \mathrm{C}(2)-\mathrm{C}(1)-\mathrm{C}(14)-\mathrm{C}(13) & -87.0(4) \\ \mathrm{C}(14)-\mathrm{C}(1)-\mathrm{C}(15)-\mathrm{C}(17) & 157.9(4) \\ \mathrm{C}(2)-\mathrm{C}(1)-\mathrm{C}(15)-\mathrm{C}(17) & 32.5(5) \\ \mathrm{C}(14)-\mathrm{C}(1)-\mathrm{C}(15)-\mathrm{C}(16) & -22.3(3) \\ \mathrm{C}(2)-\mathrm{C}(1)-\mathrm{C}(15)-\mathrm{C}(16) & -147.7(3) \\ \mathrm{C}(14)-\mathrm{O}(2)-\mathrm{C}(16)-\mathrm{O}(1) & -172.2(3) \\ \mathrm{C}(14)-\mathrm{O}(2)-\mathrm{C}(16)-\mathrm{C}(15) & 7.0(4) \\ \mathrm{C}(17)-\mathrm{C}(15)-\mathrm{C}(16)-\mathrm{O}(1) & 9.5(6) \\ \mathrm{C}(1)-\mathrm{C}(15)-\mathrm{C}(16)-\mathrm{O}(1) & -170.3(4) \\ \mathrm{C}(17)-\mathrm{C}(15)-\mathrm{C}(16)-\mathrm{O}(2) & -169.6(3) \\ \mathrm{C}(1)-\mathrm{C}(15)-\mathrm{C}(16)-\mathrm{O}(2) & 10.6(4)\end{array}$

Symmetry transformations used to generate equivalent atoms: 